\title{
CARACTERIZACIÓN DEL ESTADO TRÓFICO DE LA LAGUNA DE YAMBO MEDIANTE ANÁLISIS DE FÓSFORO
}

\section{TROPHIC STATUS SURVEY OF THE LAGUNA DE YAMBO BASED ON PHOSPHORUS ANALYSIS}

\author{
Estefanía Orquera ${ }^{1} \&$ Marcelo Cabrera ${ }^{2}$
}

Recibido: 30 de septiembre 2019 / Aceptado: 16 de diciembre 2019

DOI: 10.26807/ia.v8i1.119

Palabras clave: Índice de Estado trófico, Laguna de Yambo, Modelo de Carlson, Modelo de Aizaki, Profundidad Secchi. Keywords: Trophic State Index, Laguna de Yambo, Carlson Model, Aizaki Model, Secchi Depth.

\section{RESUMEN}

En esta investigación se realizó una caracterización de la calidad de agua de la Laguna de Yambo, ubicada en la provincia de Cotopaxi - Ecuador, a 12 km sur de Salcedo, con el fin de determinar el estado trófico de este cuerpo de agua y la detección de procesos de eutrofización. Para ello se tomaron muestras considerando un método específico de muestreo. En primera instancia se definió

1 Pontificia Universidad Católica del Ecuador, Quito, Ecuador, (*correspondencia: eiorquera@puce. edu.ec)

2 Universidad Internacional SEK, Quito, Ecuador (marcelofabian_cabrerajara@yahoo.com) 
cinco puntos de análisis en la laguna de acuerdo con coordenadas obtenidas mediante GPS en épocas distintas del año, aquí las muestras se obtuvieron a distintos niveles de profundidad y se analizaron parámetros tales como: oxígeno disuelto, conductividad, pH, temperatura y concentración de fosfatos. En la evaluación del índice de estado trófico (IET) de la Laguna de Yambo se utilizó dos criterios: el Modelo de Carlson y Modelo de Aizaki. Se efectuó una comparación entre las fórmulas propuestas en ambos modelos para determinar la concentración de fósforo total y transparencia (Profundidad Secchi) basados en los resultados obtenidos y en consideración a los intervalos de confianza para el Índice de estado Trófico. En la época seca se tiene 73,70 $\pm 3,92$ y 78,01 \pm 0,29 y en la época húmeda, 72,22 $\pm 2,44$ y 76,67 $\pm 1,41$, con lo cual se puede inferir que la Laguna de Yambo se encuentra en un estado eutrófico.

\section{ABSTRACT}

In this research a characterization of the water quality of the Laguna de Yambo, located in the province of Cotopaxi - Ecuador, $12 \mathrm{~km}$ south of Salcedo, was carried out in order to determine the trophic state of this body of water and the detection of eutrophication processes. For this research, five sample points were taken considering a specific method of sampling. First, five points of analysis was defined in different seasons of the year in the lagoon, according to coordinates obtained by GPS, here the samples were obtained at different depth levels, and parameters such as: dissolved oxygen, conductivity, $\mathrm{pH}$, temperature and phosphate concentration were analyzed. In the evaluation of the trophic state index of the Laguna de Yambo, two criteria were used, the Carlson Model and Aizaki Model and a comparison was made between the formulas proposed in both models to determine the total phosphorus concentration and transparency (Secchi Depth), based on the obtained results, and taking into account the confidence intervals for the Trophic Status Index, in the dry season there are 73.70 \pm 3.92 and $78.01 \pm 0.29$, and in the wet season, $72.22 \pm 2.44$ and $76.67 \pm 1.41$, therefore it can be inferred that the Laguna de Yambo is in a eutrophic state. 


\section{INTRODUCCIÓN}

Considerada de origen tectónico, la Laguna de Yambo, se localiza en la Provincia de Cotopaxi a $12 \mathrm{~km}$ al sur de Salcedo, vía a Ambato en la Parroquia Antonio José Holguín. Con relación a la carretera Panamericana, se encuentra unos 150 m más abajo y tiene una extensión de 1100 m de largo por 290 m de ancho. La laguna presenta una formación vegetal de matorral húmedo montano, una temperatura que oscila entre los 10 y 22 ${ }^{\circ} \mathrm{C}$ y en los últimos años se ha hecho evidente un alto desarrollo de algas y zonas turbias (Toapanta, 2017).

Considerando que existe contaminación de origen antropogénico, debido a desechos de complejos privados aledaños que son arrojados directamente sin tratamiento y la devastación de la vegetación cercana, se realizó un análisis del estado trófico de la laguna con el fin de determinar las condiciones y calidad de este cuerpo de agua (Toapanta, 2017; Rueda, 2017).

Indicadores de procesos de eutrofización son el contenido de fósforo, nitrógeno y clorofila, que estimulan el crecimiento del fitoplancton, especialmente de algas, y el análisis de la profundidad Secchi (Álamo et al., 2013).

Considerando estos parámetros se analiza los diversos estados tróficos que las fuentes hídricas pueden presentar. Así, se tiene el estado oligotrófico, donde las fuentes de agua presentan alta transparencia, lo que permite el paso de la luz hasta el fondo, con poca cantidad de nutrientes y sustancias húmicas, lo que resulta en una baja producción vegetal. (López Martínez \& Madroñero Palacios, 2015; Fraile, Pozo, Ciencias, \& Vasco, 1994) y, el estado mesotrófico que muestra cuerpos de agua con características intermedias entre estados extremos de concentración de nutrientes y biomasa, un oligotrófico (baja concentración) y un eutrófico (alta concentración) (CEPIS, 1989).

Se puede apreciar condiciones de eutrofización en fuentes de agua cuando se tiene niveles de nutrientes particularmente altos, con producción vegetal excesiva y estancamiento de agua, situación prácticamente 
irreversible por la abundancia anormal de nutrientes. Situaciones de extrema eutrofia -donde la luz es el factor medioambiental dominante de control de crecimiento- ocurren en estados hipertróficos (Resendiz, 2012).

En la Tabla 1 se muestran las cuatro categorías del Índice de Estado Trófico de Carlson (1977) o TSI (Trophic state index).

Tabla 1. Índice de Estado Trófico (TSI)

\begin{tabular}{cc}
\hline & TSI \\
\hline Oligotrófico & Mesotrófico \\
TSI $<30$ & $30<$ TSI $<60$ \\
\hline Eutrófico & Hipertrófico \\
$60<\mathrm{TSI}<90$ & $90<\mathrm{TSI}<100$ \\
\hline
\end{tabular}

A pesar de que en otros estudios se ha señalado que la laguna es de tipo Eutrófica, como aquellos Ilevados a cabo por Toapanta (2017) y Rueda (2017), la presente investigación busca caracterizar el estado trófico de la Laguna de Yambo. El lugar se considera un potencial sitio turístico, señalado en trabajos como los de Villaroel y Ortiz (2015) y Torres (2019). Con el incremento de visitantes, construcción de localidades para actividades recreativas, entre otros, se pueden acarrear impactos medioambientales, razón por la que monitoreos ambientales continuos de este cuerpo de agua fomentarían acciones para su preservación.

\section{MATERIALES Y MÉTODOS}

Para la evaluación de la eutrofización de la Laguna de Yambo, se llevaron a cabo dos salidas de campo, en dos épocas distintas del año. La primera salida fue el 15 de junio de 2019 y la segunda el 2 de noviembre de 2019, que coinciden con la época seca, de junio a septiembre, y época húmeda, septiembre a mayo, de acuerdo a información de la estación meteorológica Rumipamba-Salcedo del Anuario 2017 del INAMHI (Inamhi, 2017). Se usó un GPS Garmin Etrex H, donde se tomaron las coordenadas y se seleccionó los puntos mostrados en la Tabla 2. 
Tabla 2. Puntos de Muestreo

\begin{tabular}{ccc}
\hline PUNTO & \multicolumn{2}{c}{$\begin{array}{c}\text { Coordenadas (UTM): } \\
\text { Zona 17, hemisferio S }\end{array}$} \\
\cline { 2 - 3 } & $\mathbf{X}$ & \multicolumn{1}{c}{ Y } \\
\hline A & 768296,2 & 9878356,5 \\
B & 768127,1 & 9878356,5 \\
C & 768390,8 & 9878158,0 \\
D & 768487,7 & 9877951,5 \\
E & 768651,1 & 9877681,2 \\
\hline
\end{tabular}

En la Figura 1, se reportan los puntos indicados.

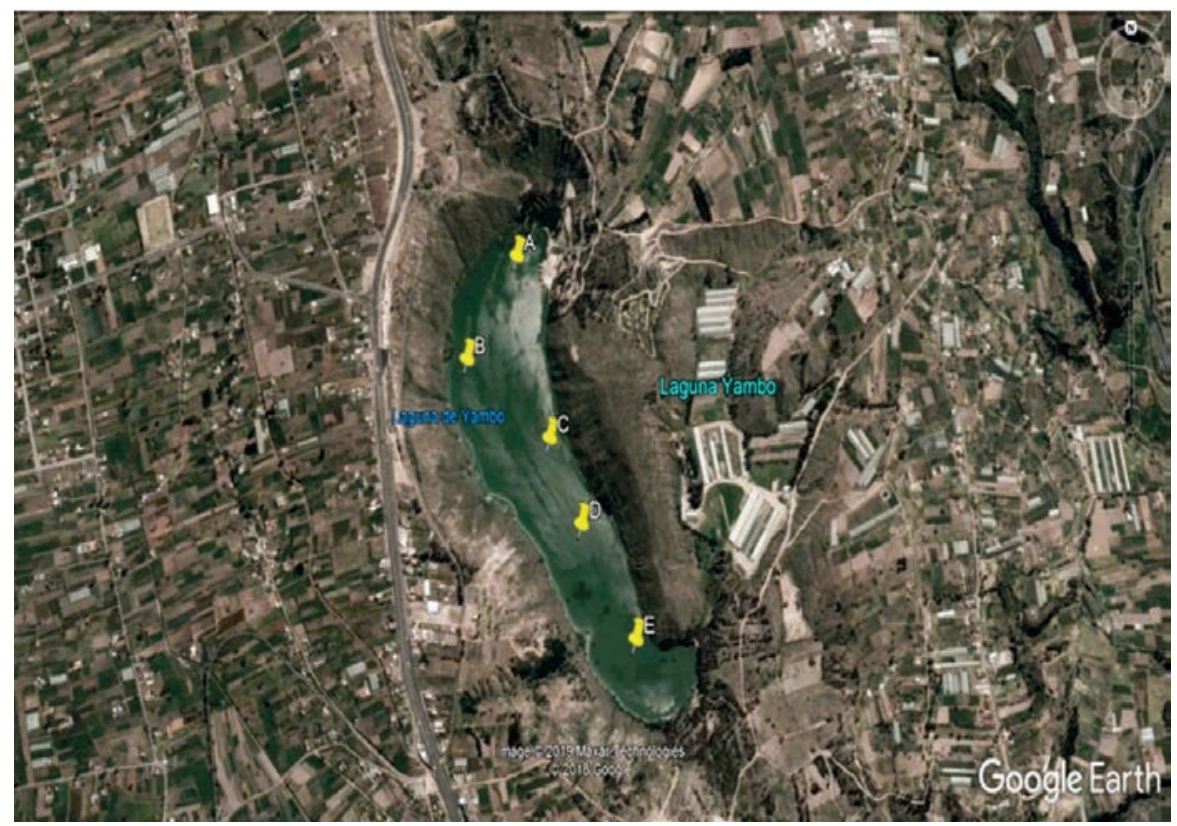

Figura 1. Ubicación puntos de muestreo

Con una jarra se tomó una muestra de $1 \mathrm{~L}$ de agua superficial $y$, con la ayuda de un equipo para toma de muestras de agua tipo Van Dorn, se tomó una muestra de 1 litro de agua a 2 metros de profundidad. Ambas 
muestras fueron recolectadas y mezcladas en un recipiente plástico previamente homogenizado. Así se formó una muestra compuesta de 2 litros en cada punto de muestreo (NTE INEN 2117:2013, 2013).

En estos puntos se determinó la profundidad Secchi. La muestra compuesta recolectada en cada punto fue almacenada bajo sombra. Una vez en el muelle, se analizó oxígeno disuelto, conductividad, $\mathrm{pH}$ y temperatura, mediante un equipo multiparámetro HACH HQ40D. Se determinó la concentración de fosfatos con el Espectrofotómetro HACH DR/850. Se escogió como método estándar de análisis de agua y aguas residuales el Método PhosVer 3 (ácido ascórbico), Fósforo Reactivo (0 a 2,50 mg / L $\left.\mathrm{PO}_{4}{ }^{3-}\right)$ que requirió un tiempo de reacción de 3 minutos.

La determinación del índice de estado trófico de la Laguna de Yambo se realizó tomando como referencia los trabajos investigativos de López et al. (2015), y Fraile et al. (1994), evaluando los siguientes dos criterios:

\section{Índice de Carlson o índice de estado trófico (IET):}

El modelo de Carlson propuesto en 1977 emplea el Disco de Secchi para determinar la transparencia del agua y, en conjunto con la concentración de fósforo total, se obtiene la ecuación que determina el Índice de Estado Trófico IET.

\section{Fósforo total (PT):}

La ecuación que relaciona la cantidad de fósforo total con el índice de estado trófico es:

$$
I E T_{P T}=10 *\left(6-\frac{\ln \left(\frac{65}{P T}\right)}{\ln (2)}\right)
$$

PT: concentración de fósforo en $\square \mathrm{g} / \mathrm{L}$.

\section{Profundidad Secchi:}

La ecuación que relaciona la transparencia con el índice de estado trófico es:

$$
I E T_{S e c}=10 *\left(6-\frac{\ln (\mathrm{Sec})}{\ln (2)}\right)
$$

Sec es el promedio de la altura Secchi tomada con el disco que lleva el mismo nombre, en metros. 


\section{Modelo de Aizaki:}

El modelo de Aizaki et al (1981) resulta una modificación al IET propuesto por Carlson donde incluye coeficientes específicos para los elementos que integran la ecuación de estado trófico:

\section{Fósforo Total (PT):}

La ecuación que relaciona el fósforo total con el índice de estado trófico es:

$$
I E T_{P T}=10 *\left(2,46+\frac{6,68+1,15 * \ln (P T)}{\ln (2,5)}\right)
$$

PT: concentración de fósforo en $\mathrm{mg} / \mathrm{L}$.

\section{Profundidad Secchi:}

La ecuación que relaciona la transparencia con el índice de estado trófico es:

$$
I E T_{S e c}=10 *\left(2,46+\frac{3,76-1,57 * \ln (D s)}{\ln (2,5)}\right)
$$

DS: promedio altura Secchi en metros.

En la Tabla 3 se muestran los valores bibliográficos tomados como referencia para la determinación del IET.

\begin{tabular}{|c|c|c|c|}
\hline Estado de Eutrofia & TSI & Ds (m) & $\mathrm{Pt}\left(\mathrm{mg} / \mathrm{m}^{3}\right)$ \\
\hline $\begin{array}{c}\text { Oligotrófico } \\
\text { TSI }<30\end{array}$ & $\begin{array}{c}0 \\
10 \\
20 \\
30\end{array}$ & $\begin{array}{c}64 \\
32 \\
16 \\
8\end{array}$ & $\begin{array}{c}0,75 \\
1,5 \\
3 \\
6\end{array}$ \\
\hline $\begin{array}{l}\text { Mesotrófico } \\
30<\text { TSI < } 60\end{array}$ & $\begin{array}{l}40 \\
50 \\
60\end{array}$ & $\begin{array}{l}4 \\
2 \\
1\end{array}$ & $\begin{array}{l}12 \\
24 \\
48\end{array}$ \\
\hline $\begin{array}{c}\text { Eutrófico } \\
60<\mathrm{TSI}<90\end{array}$ & $\begin{array}{l}70 \\
80 \\
90\end{array}$ & $\begin{array}{c}0,5 \\
0,25 \\
0,12\end{array}$ & $\begin{array}{c}96 \\
192 \\
384\end{array}$ \\
\hline $\begin{array}{c}\text { Hipereutrófico } \\
90<\mathrm{TSI}<100\end{array}$ & $1^{00}$ & 0,06 & $768^{*}$ \\
\hline
\end{tabular}

Tabla 3. Escala de valores del estado trófico en los cuerpos de agua

Modificado de Carlson (1977; 1980) 


\section{RESULTADOS}

En la Tabla 4 y Tabla 5 se presentan los resultados de los parámetros fisicoquímicos de la muestra tomada en los puntos de muestreo de las fechas indicadas anteriormente:

Tabla 4. Parámetros fisicoquímicos 15 jun-2019

\begin{tabular}{ccccc}
\hline Punto & $\begin{array}{c}\text { Tempera- } \\
\text { tura } \\
\left({ }^{\circ} \mathrm{C}\right)\end{array}$ & $\begin{array}{c}\mathrm{pH} \\
-\end{array}$ & $\begin{array}{c}\text { Conducti- } \\
\text { vidad } \\
\mu \mathrm{S} / \mathrm{cm}\end{array}$ & $\begin{array}{c}\mathrm{O}_{2} \\
\text { disuelto } \\
\mathrm{mg} / \mathrm{L}\end{array}$ \\
\hline A & 19,4 & 8,93 & 2610 & 6,95 \\
B & 19,5 & 8,81 & 2489 & 6,88 \\
C & 19,7 & 8,87 & 2530 & 6,82 \\
D & 19,9 & 8,80 & 2356 & 6,80 \\
E & 19,7 & 8,90 & 2651 & 6,85 \\
\hline
\end{tabular}

Tabla 5. Parámetros fisicoquímicos 2 nov-2019

\begin{tabular}{ccccc}
\hline Punto & $\begin{array}{c}\text { Tempera- } \\
\text { tura } \\
\left({ }^{\circ} \mathrm{C}\right)\end{array}$ & $\begin{array}{c}\mathrm{pH} \\
--\end{array} \begin{array}{c}\text { Conducti- } \\
\text { vidad } \\
\mu \mathrm{S} / \mathrm{cm}\end{array}$ & $\begin{array}{c}\mathrm{O}_{2} \\
\text { disuelto } \\
\mathrm{mg} / \mathrm{L}\end{array}$ \\
\hline A & 18,1 & 8,71 & 2190 & 6,73 \\
B & 18,0 & 8,78 & 2278 & 6,79 \\
C & 17,9 & 8,81 & 2474 & 6,70 \\
D & 18,0 & 8,77 & 2389 & 6,71 \\
E & 18,2 & 8,86 & 2463 & 6,81 \\
\hline
\end{tabular}

Para el caso de la altura Secchi, fosfatos y fósforo, se muestran en la Tabla 6 y Tabla 7 los valores para las dos fechas de medición
Tabla 6. Altura Secchi, Fosfatos y fósforo, 15 jun- 2019

\begin{tabular}{cccc}
\hline Punto & $\begin{array}{c}\text { Altura } \\
\text { Secchi } \\
\mathrm{m}\end{array}$ & $\begin{array}{c}\text { Fosfatos } \\
\mathrm{mg} / \mathrm{L}\end{array}$ & $\begin{array}{c}\text { *Fósforo } \\
\mu \mathrm{g} / \mathrm{L}\end{array}$ \\
\hline A & 0,476 & 0,62 & 202,21 \\
B & 0,481 & 0,61 & 198,95 \\
C & 0,485 & 0,67 & 218,52 \\
D & 0,488 & 0,65 & 211,99 \\
E & 0,479 & 0,66 & 215,25 \\
\hline
\end{tabular}

Tabla 7. Altura Secchi, Fosfatos y fósforo, 2 nov- 2019

\begin{tabular}{cccc}
\hline Punto & $\begin{array}{c}\text { Altura } \\
\text { Secchi } \\
\mathrm{m}\end{array}$ & $\begin{array}{c}\text { Fosfatos } \\
\mathrm{mg} / \mathrm{L}\end{array}$ & $\begin{array}{c}\text { *Fósforo } \\
\mu \mathrm{g} / \mathrm{L}\end{array}$ \\
\hline A & 0,490 & 0,56 & 182,64 \\
B & 0,494 & 0,53 & 172,86 \\
C & 0,491 & 0,50 & 163,07 \\
D & 0,489 & 0,59 & 192,42 \\
E & 0,493 & 0,49 & 159,81 \\
\hline * Calculado sobre la base de relaciones estequiométricas a \\
partir del dato de concentración de fosfatos
\end{tabular}

Los resultados obtenidos del IET empleando ambos modelos, para el 15 de junio de 2019 se muestran en la Tabla 8 y en la Tabla 9: 
Tabla 8. Índice de estado Trófico según Modelo Carlson (15 jun 2019)

\begin{tabular}{ccccc}
\hline & \multicolumn{4}{c}{ Modelo Carlson } \\
\cline { 2 - 5 } Punto & IET $_{\text {PT }}$ & IET $_{\text {Sec }}$ & IEC $_{\text {Prom }}$ & $\begin{array}{r}\text { IEC }_{\text {Desv. }} \\
\text { Std }\end{array}$ \\
\hline A & 76,37 & 70,71 & 73,54 & 4,00 \\
B & 76,14 & 70,56 & 73,35 & 3,95 \\
C & 77,49 & 70,44 & 73,97 & 4,99 \\
D & 77,06 & 70,35 & 73,70 & 4,74 \\
E & 77,28 & 70,62 & 73,95 & 4,71 \\
\hline
\end{tabular}

PROMEDIO 73,70 $\pm 4,48$

Intervalo de Confianza 3,92

Tabla 9. Índice de estado Trófico según Modelo Aizaki (15 jun 2019)

\begin{tabular}{|c|c|c|c|c|}
\hline \multirow[b]{2}{*}{ Punto } & \multirow[b]{2}{*}{$\mathrm{IET}_{\mathrm{PT}}$} & \multicolumn{3}{|c|}{ Modelo Aizaki } \\
\hline & & $\mathrm{IET}_{\mathrm{Sec}}$ & $\begin{array}{l}\text { IEA } \\
\text { Prom }\end{array}$ & $\mathrm{IEC}_{\text {Std }}$ \\
\hline A & 77,44 & 78,35 & 77,90 & 0,65 \\
\hline B & 77,24 & 78,18 & 77,71 & 0,66 \\
\hline C & 78,41 & 78,03 & 78,22 & 0,27 \\
\hline D & 78,03 & 77,93 & 77,98 & 0,08 \\
\hline E & 78,23 & 78,25 & 78,24 & 0,01 \\
\hline \multicolumn{2}{|c|}{ PROMEDIO } & \multicolumn{2}{|c|}{$78,01 \pm 0,33$} & \\
\hline Inter & alo de & onfianzc & 0,29 & \\
\hline
\end{tabular}

Las determinaciones de IET con ambos modelos del 2 de noviembre de 2019 se muestran en la Tabla 10 y en la Tabla 11:
Tabla 10. Índice de estado Trófico según Modelo Carlson (2 nov- 2019)

\begin{tabular}{ccccc}
\hline & \multicolumn{4}{c}{ Modelo Carlson } \\
\cline { 2 - 5 } Punto & IET $_{\text {PT }}$ & IET $_{\text {Sec }}$ & $\begin{array}{c}\text { IEC } \\
\text { Prom }\end{array}$ & $\begin{array}{c}\text { IEC }_{\text {Sesv. }} \\
\text { Std }\end{array}$ \\
\hline A & 74,90 & 70,29 & 72,60 & 3,26 \\
B & 74,11 & 70,17 & 72,14 & 2,78 \\
C & 73,27 & 70,26 & 71,77 & 2,13 \\
D & 75,66 & 70,32 & 72,99 & 3,77 \\
E & 72,98 & 70,20 & 71,59 & 1,96 \\
\hline PROMEDIO $72,22 \pm 2,78$ \\
\hline \multicolumn{5}{l}{ Intervalo de Confianza } \\
\hline
\end{tabular}

Tabla 11. Índice de estado Trófico según Modelo Aizaki (noviembre 2019)

\begin{tabular}{|c|c|c|c|c|}
\hline \multirow[b]{2}{*}{ Punto } & \multicolumn{4}{|c|}{ Modelo Aizaki } \\
\hline & $\mathrm{IET}_{\mathrm{PT}}$ & $\mathrm{IET}_{\mathrm{Sec}}$ & IEA $_{\text {prom }}$ & $\begin{array}{l}\mathrm{IEC}_{\text {Desv }} \\
\text { Std }\end{array}$ \\
\hline A & 76,16 & 77,86 & 77,01 & 1,20 \\
\hline B & 75,47 & 77,72 & 76,60 & 1,59 \\
\hline c & 74,74 & 77,82 & 76,28 & 2,18 \\
\hline D & 76,82 & 77,89 & 77,36 & 0,76 \\
\hline E & 74,49 & 77,75 & 76,12 & 2,31 \\
\hline \multicolumn{5}{|c|}{ PROMEDIO $76,67 \pm 1,61$} \\
\hline Inte & , & anza & 1,41 & \\
\hline
\end{tabular}

De acuerdo con los resultados obtenidos, y tomando en consideración los intervalos de confianza para el Índice de estado Trófico, en la época seca se tiene $73,70 \pm 3,92$ y 78,01 \pm 0,29 y en la época húmeda, 72,22 \pm 
2,44 y $76,67 \pm 1,41$. Con esto se puede inferir que la Laguna de
Yambo se encuentra en un estado eutrófico.

\section{DISCUSIÓN}

Esta investigación permitió determinar, a partir de análisis de parámetros fisicoquímicos en ambas épocas del año del estudio, que el $\mathrm{pH}$ supera un valor de 8,71 en cada punto de monitoreo, lo cual significa que existe una disminución de acidez, de acuerdo con lo señalado por Toapanta (2017), probablemente por la presencia y proliferación de algas que emplean $\mathrm{CO}_{2}$ para su crecimiento y producción.

La inspección in situ mostró, en los puntos A, C y E la existencia de zonas pobladas, construcción de lugares de esparcimiento y con ello efluentes enviados directamente a la laguna sin tratamiento, los cuales pueden ser considerados fuentes de contaminación y a su vez causas de procesos de eutrofización.

En la época seca se aprecia una mayor concentración de fosfatos que en la época húmeda, lo cual permite inferir posibles diluciones; sin embargo, no son representativas, pues
Ios IET son superiores a 70, con lo que se deduce procesos de eutrofización de la Laguna de Yambo.

Como se menciona en la investigación de Jarosiewicz et al. (2012), en la determinación del estado trófico actual de ocho lagos de Polonia empleando el índice de estado trófico de Carlson (IET) con el cálculo de fósforo total (TP) y otros parámetros como Clorofila a y Nitrógeno, indica que los lagos presentan estados entre mesotróficos y eutróficos. Esta determinación se ve confirmada principalmente por el índice derivado de la concentración de fósforo total. Los valores de TP en los lagos analizados son más altos que los valores de los índices calculados en base a las otras variables con lo cual el estado trófico está asociado con los valores más altos de TP en todos los lagos investigados. Así se tiene: el Lago Jelén con un IET (TP) de 64,8 y el Lago Rybiec con 79, el Niezabyszewo con 77,8, Czarne con 72,5, Chotkowskie con 79,1, Obłeze con 78,1, Jasień S 75,9, 
Jasień N 73,3. Con estos resultados, el estudio señala que altas concentraciones de fósforo se ven asociados con un inadecuado o no sistema existente de aguas residuales, principalmente en zonas rurales, como lo que ocurre con el Lago Rybiec y Obłeze, que carecen de plantas de tratamiento de aguas residuales en sus inmediaciones. En consecuencia, existe tendencia al crecimiento excesivo de algas, un enturbiamiento de estos lagos y una reducción progresiva de la biodiversidad lacustre (Jarosiewicz, Ficek, \& Zapadka, 2012).

Estudios realizados en Colombia por López y Madroñero (2015) para la determinación del estado trófico de un lago tropical de alta montaña, la Laguna de La Cocha mediante el Índice de estado Trófico de Carlson durante un año de evaluación, 2013, determinó un estado oligotrófico, debido a la carencia de nutrientes y contaminación por causa de actividades antropogénicas en este tipo de lagunas de altura. La laguna de La Cocha se encuentra ubicada a unos 20 km de Pasto, a 2.800 metros sobre el nivel del mar y rodeada por montañas (López Martínez \& Madroñero Palacios, 2015).
Por otra parte, en el estudio de Li et al. (2010), para evaluar el estado trófico del agua en los humedales del parque de Xixi en China también se empleó el Índice de estado Trófico de Carlson, el cual evidenció un estado de eutrofización. En el análisis de resultados se determinó que esta condición puede estar asociada a que este cuerpo de agua recibe gran cantidad de nutrientes por su cercanía a cultivos y ciudades que envían sus aguas sin tratamiento previo (Li et al., 2010).

En este sentido, los resultados obtenidos para la Laguna de Yambo son consistentes con los establecido en las investigaciones de Jarosiewicz et al. (2012), Li et al. (2010), Toapanta (2017) y Rueda (2017) quienes indican que un estado eutrófico de los cuerpos de agua se relaciona con la cantidad de nutrientes introducidos producto de actividades antropogénicas en los alrededores, lo que trae como consecuencia el probable crecimiento acelerado de biomasa y pérdida de biodiversidad de la Laguna. Se complementan con lo dicho por López y Madroñero (2015), que manifiestan que los cuerpos hídricos con poca o ninguna contaminación 
a causa de la intervención humana presentan estados oligotróficos, que permiten la conservación de sus eco- sistemas y de la variedad de seres vivos.

\section{CONCLUSIONES}

De acuerdo con los resultados de Índice de estado Trófico, empleando los modelos de Carlson y de Aizaki, en la época seca se tienen $73,70 \pm$ 3,92 y 78,01 $\pm 0,29$ y en la época húmeda, 72,22 $\pm 2,44$ y 76,67 \pm 1,41 , valores consistentes durante todo el año de estado eutrófico de la Laguna de Yambo.

Los puntos de muestreo A, C y E revelan Índices de estado Trófico superiores a los del resto de puntos, mismos que en sus cercanías indican zonas pobladas, con lo cual se podría inferir que existen efluentes enviados directamente a la Laguna de Yambo sin tratamiento previo que favorecen estados eutróficos.

La determinación de la concentración de clorofila podría fortalecer los resultados de la presente investigación ya que es un indicativo de la existencia o inexistencia de plantas acuáticas que aportan con oxígeno disuelto al agua de la laguna y se podría sugerir para próximos estudios.

\section{LISTA DE REFERENCIAS}

Álamo, B., Loza, S., Sánchez, M., Montalvo, J. F., García, I., Reyes, T., \& Carmenate, M. (2013). Evaluación del estado trófico de seis bahías interiores del Archipiélago Sabana- Camaguey, Cuba, mediante el empleo de clorofila-a como bioindicador Assessment of the trophic state of six inner bays in Sabana-Camagüey Archipelago, Cuba, using chlorophyll-. Serie Oceanológica No, 13(13), 9-21.

CEPIS. (1989). Curso Basico Sobre Eutroficación. 
Fraile, H., Pozo, E. O. J., Ciencias, F., \& Vasco, P. (1994). Evaluación del Estado Trófico y Comparación de Modelos relativos al Fósforo en los embalses de Cernadilla Y Valparaíso (Río Tera, Zamora). Water.

Inamhi. (2017). Boletín Climatologico Decadal. Retrieved from http://www.serviciometeorologico.gob.ec/docum_institucion/anuarios/meteorologicos/Am_2013.pdf

Jarosiewicz, A., Ficek, D., \& Zapadka, T. (2012). Eutrophication parameters and Carlson-type trophic state indices in selected Pomeranian lakes. Limnological Review, 11(1), 15-23. https://doi.org/10.2478/v10194-011-0023-3

Li, Y., Liu, H., Zheng, N., \& Cao, X. (2010). Analysis of trophic status and its influence factors of different water body types in Xixi National Wetland Park, China. Procedia Environmental Sciences, 2(5), 768-780. https://doi.org/10.1016/j.proenv. 2010.10.088

López Martínez, M. L., \& Madroñero Palacios, S. M. (2015). Estado trófico de un lago tropical de alta montaña: Caso Laguna de la Cocha. Ciencia e Ingeniería Neogranadina, 25(2), 21. https://doi.org/10.18359/rcin.1430

NTE INEN 2117:2013. (2013). NORMA TÉCNICA ECUATORIANA NTE INEN 266 : 2013 Primera revisión, First Edit, 7-12.

Resendiz Cruz, J. A. (2012). Evaluación de la calidad del agua en lagos y embalses, 532.

Rueda, G. (2017). Composición Espacio-Temporal De Comunidades De Aves Acuáticas En Seis Lagunas Altoandinas Del Ecuador. Universidad Central del Ecuador. Retrieved from http://www.dspace.uce.edu.ec/bitstream/25000/12997/1/T-UCE0016-012.pdf

Toapanta, M. (2017). Determinación del Estado Trófico de la lagunade Yambo a través de la cuantificación de clorofila "A." Universidad Central del Ecuador. Universidad Central del Ecuador. Retrieved from http://www.dspace.uce.edu.ec/bitstream/ 25000/13141/1/T-UCE-0012-39.pdf 